\title{
ENTIRE FUNCTIONS WITH TWO LINEARLY DISTRIBUTED VALUES
}

\author{
I. N. BAKER
}

In a series of papers $[2,3,4] \mathrm{T}$. Kobayashi has given some interesting characterisations of the exponential function by its property of having its $a$-points collinear on a line $\lambda(a)$ for several values of $a$. In [4] he proved

Theorem A. Let $G$ be a transcendental entire function of finite lower order Assume that the zero points of $G$ lie on the line $\operatorname{Re} z=0$ and that the one-points lie on $\operatorname{Re} z=1$. Then

$$
G(z)=P(\exp C z)
$$

where $P$ is a polynomial and $C$ a non-zero real constant.

Of course there are restrictions on the polynomials which can occur in Theorem $\mathrm{A}$ and the possible forms are given in [4].

Kobayashi asks whether Theorem A still holds if the assumption that $G$ has finite order be omitted from the hypotheses. We prove the

Theorem. Let $f$ be a transcendental entire function such that all the zeros of $f$ lie on $\operatorname{Re} z=0$ and all the one-points on $\operatorname{Re} z=1$. Then $f$ has finite order, so by Theorem A there exist a polynomial $P$ and a non-zero real constant $C$ such that

$$
f(z)=P(\exp C z) \text {. }
$$

The proof depends on a recent result of J. Miles (Lemma 6) which states that if an entire function has both infinite order and real zeros then the zeros are in a certain sense scarce. Applying this to $f(-i z)$ and $f(1-i z)-1$ and using Nevanlinna's second fundamental theorem gives the result after a number of subsidiary points have been checked.

The proof follows in six lemmas and a concluding section.

Lemma 1. Let $g$ be analytic in $H: \operatorname{Im} z>0$ and omit the values 0 and 1 in $H$. Then there exists a constant $K=K(g)$ such that

$$
\log \left|g\left(r e^{i \theta}\right)\right|<K r /(\sin \theta), \quad r>1,0<\theta<\pi .
$$

Proof. The map $z=\varphi(t)=i(1+t) /(1-t)$ maps the disc $D:|t|<1$ to the half-plane $H$. Applying Schottky's theorem to $g(\varphi(t))$ we obtain

$$
|g(\varphi(t))|<\exp \{K /(1-|t|)\}
$$


Using

we have

$$
1-|t|^{2}=4 r \sin \theta /\left\{r^{2}+2 r \sin \theta+1\right\}, \quad z=r e^{i \theta}
$$

and

$$
1-|t|>2 r \sin \theta /\left\{r^{2}+2 r \sin \theta+1\right\}, \quad|t|<1
$$

$$
\log \left|g\left(r e^{i \theta}\right)\right|<K(1+r)^{2} /(2 r \sin \theta)<K r /(\sin \theta) .
$$

Lemma 2. Let $g$ be analytic in the strip $S: 0<\operatorname{Im} z<1$ and omit the values 0 and 1 there. Then there exists a constant $K=K(g)$ such that

$$
\log |g(x+i y)|<K e^{\pi|x|} /(\sin \pi y), \quad 0<y<1,-\infty<x<\infty .
$$

Proof. Putting $w=e^{\pi z}$ which maps $S$ onto the half-plane $H: \operatorname{Im} w>0$, the result follows from Lemma 1 for $x>0$. For $x<0$ the result follows by symmetry.

Le mma 3. Suppose $f$ is entire and that all the zeros of $f$ are real and all the onepoints have imaginary part one. Then there is a constant $A$ such that

$$
T(r)=T(r, f)<A r^{-4} e^{4 \pi r}
$$

for all sufficiently large $r$.

Remark. We assume without explanation the standard notations of Nevanlinna theory.

Proof. The result of Lemma 1 shows that $f$ is of order one and exponential type in any angle which is either strictly interior to $\operatorname{Im} z>0$ or to $\operatorname{Im} z<0$. If the lower order $\mu=\underline{\lim }_{r \rightarrow \infty}(\log T(r)) /(\log r)<\infty$ it follows from the PhragmènLindelöf principle that $f$ has at most order one in the plane and the assertion of the lemma holds.

Thus we may assume that $\mu=\infty$ so that $T(r) \rightarrow \infty$ faster than any power of $r$. Put $\eta=\sin ^{-1}(1 / r)$ and $\delta=\{T(r)\}^{-1 / 2}$. Split the range of integration in

$$
T(r)=\frac{1}{2 \pi} \int_{0}^{2 \pi} \log { }^{+}\left|f\left(r e^{i \theta}\right)\right| d \theta
$$

at $\pm \delta, \eta \pm \mathcal{J}, \pi-\eta \pm \delta$ and $\pi \pm \delta$. In the intervals $[-\delta, \delta],[\eta-\delta, \eta+\delta]$, $[\pi-\eta-\delta, \pi-\eta+\delta]$ and $[\pi-\delta, \pi+\delta]$ we put

$$
\log +\left|f\left(r e^{i \theta}\right)\right| \leqq \log M(r, f),
$$

and in the remaining intervals use the estimates from Lemmas 1 and 2 . It follows that there are constants $K, K^{\prime}, P$ and $Q$ such that for sufficiently large $r$

$$
\begin{aligned}
T(r) & <\frac{K r}{\sin \delta}+\frac{K^{\prime} e^{\pi r} \eta}{\sin (\pi r \delta)}+\frac{4 \delta}{\pi} \log M(r, f) \\
& <\frac{P r}{\delta}+\frac{Q e^{\pi r}}{r^{2} \delta}+\frac{4 \delta}{\pi} \log M(r, f) .
\end{aligned}
$$


Taking $R=r+\varphi$ where $\varphi=r / \log T(r)$ we have

$$
\log M(r, f) \leqq \frac{R+r}{R-r} \cdot T(R)>\frac{3 r}{\varphi} T\left(r+\frac{r}{T(r)}\right) .
$$

A lemma of Borel [1] states that for any increasing function $V(r)$ which is continuous in $r>r_{0}$ and such that $V(r) \rightarrow \infty$ as $r \rightarrow \infty$ and for any $\varepsilon>0$

$$
V\left(r+\frac{r}{\log V(r)}\right) \leqq V(r)^{1+\varepsilon}
$$

holds outside a set of finite logarithmic measure. Taking $V=T$ and $1 / 2>\varepsilon>0$, (4) and (5) show that outside a set $E$ of finite logarithmic measure in $r>1$

$$
T(r)<\operatorname{Pr} \delta^{-1}+Q r^{-2} \delta^{-1} e^{\pi r}+12 \delta r T(r)^{1+\varepsilon} /(\pi \varphi),
$$

or putting in the values of $\delta$ and $\varphi$

$$
T(r)<\operatorname{Pr} T(r)^{1 / 2}+Q r^{-2} e^{\pi r} T(r)^{1 / 2}+(12 / \pi)(\log T(r)) T(r)^{1 / 2+\varepsilon} .
$$

For $0<\varepsilon<\varepsilon^{\prime}<1 / 2$ we have

$$
\log T(r)=o\left((T(r))^{\varepsilon^{\prime}-\varepsilon}\right) \quad(r \rightarrow \infty)
$$

and so

$$
(1-o(1)) T(r)^{1 / 2}<2 Q r^{-2} e^{\pi r}
$$

as $r \rightarrow \infty$ outside $E$. Hence for large $r$ outside $E$

$$
T(r)<5 Q^{2} r^{-4} e^{2 \pi r} .
$$

Since $E$ has finite logarithmic measure there exists $r_{0}$ such that for any $r>r_{0}$ there are $s \notin E, t \notin E, s<r<t<2 s$, while (6) holds for $r=s$ and $r=t$. Thus

$$
T(r)<T(t)<5 Q^{2} t^{-4} e^{2 \pi t}<5 Q^{2} r^{-4} e^{4 \pi r},
$$

so that (2) holds with $A=5 Q^{2}$.

Lemma 4. Suppose the increasing continuous function $V(r)$ satisfies $V(r)<e^{A r}$ for some constant $A$, at least for all $r \geqq r_{0}>0$. Suppose also that $V(r) \rightarrow \infty$ as $r \rightarrow \infty$. Then

$$
V\left(r+r^{-1}\right)<2 V(r)
$$

holds outside a set of finite logarithmic measure in $\left[r_{0}, \infty\right)$.

Proof. Suppose the assertion is not true. Then there is a first $r_{1} \geqq r_{0}$ where (7) fails. Define $r_{1}^{*}=r_{1}+r_{1}^{-1}$. Then

$$
V\left(r_{1}^{*}\right) \geqq 2 V\left(r_{1}\right) .
$$

Denote the interval $\left[r_{1}, r_{1}^{*}\right]$ by $I_{1}$.

Now proceed inductively. Assuming $r_{n}, r_{n}^{*}=r_{n}+r_{n}^{-1}$ and $I_{n}=\left[r_{n}, r_{n}^{*}\right]$ have been constructed, let $r_{n+1}$ be the first $r \geqq r_{n}^{*}$ at which (7) fails, i.e. $V\left(r_{n+1}^{*}\right) \geqq 2 V\left(r_{n+1}\right)$. 
Such an $r_{n+1}$ exists since we are supposing that (7) fails for a set of infinite logarithmic measure. Thus there is an infinite sequence $r_{n}, n=1,2, \ldots$, and we have

$$
r_{n}<r_{n}^{*} \leqq r_{n+1}<r_{n+1}^{*} \text {. }
$$

From this it follows that $r_{n} \rightarrow \infty$ for otherwise we would have $\lim r_{n}=\lim r_{n+1}=$ $\lim r_{n}^{*}=$ finite and thence $\lim r_{n}^{-1}=0$ which gives a contradiction. Further, the inequality (7) holds in the complement of $F=\bigcup_{n=1}^{\infty} I_{n}$.

The logarithmic measure of $I_{n}$ is $\log \left(1+r_{n}^{-2}\right)<r_{n}^{-2}$. Since the logarithmic measure of $F$ is infinite $\sum r_{n}^{-2}=\infty$. Take any constant $B$ such that $B \log 2>A$. There must be arbitrarily large $n$ such that $r_{n}^{-2} \geqq B^{2} n^{-2}$, that is such that $n \geqq B r_{n}$. For any $n$

$$
V\left(r_{n}\right) \geqq V\left(r_{n-1}^{*}\right) \geqq 2 V\left(r_{n-1}\right) \geqq \lambda 2^{n},
$$

where $\lambda=V\left(r_{1}\right) / 2$. For infinitely many $n$ we have in addition that $n \geqq B r_{n}$ so that

$$
V\left(r_{n}\right)>\lambda \exp \left(r_{n} B \log 2\right) \text {. }
$$

Since $r_{n} \rightarrow \infty$ and $B \log 2>A$ this contradicts the hypothesis that $V(r)<e^{A r}$, $r>r_{0}$. Thus the lemma is established.

Lemma 5. Suppose $f$ satisfies the assumptions of Lemma 3 and let $g$ be defined by $g(z)=f(i+z)$.

Then

$$
T(r, g)<8 T(r, f)
$$

outside a set $E_{1} \subset[1, \infty)$ of finite logarithmic measure.

Proof. We can assume $f$ transcendental. By Nevanlinna's second fundamental theorem

$$
T(r, g) \leqq N(r, 0, g)+N(r, 1, g)+S(r)
$$

where $S(r)=O\{\log r+\log T(r, g)\}$ as $r \rightarrow \infty$ outside a set $H$ of finite measure. Now

so that

$$
\begin{aligned}
& n(r, 0, g) \leqq n(r, 0, f), \\
& n(r, 1, g) \leqq n\left(\left(1+r^{2}\right)^{1 / 2}, 1, f\right),
\end{aligned}
$$

$$
N(r, 0, g) \leqq N(r, 0, f)+O(\log r)
$$

and

$$
\begin{aligned}
N(r, 1, g) & =O(\log r)+\int_{1}^{r} n(t, 1, g) \frac{d t}{t} \\
& \leqq O(\log r)+\int_{\sqrt{2}}^{\sqrt{\left(1+r^{2}\right)}} \frac{n(u, 1, f)}{u^{2}-1} u d u \\
& \leqq O(\log r)+2 N\left(\left(1+r^{2}\right)^{1 / 2}, 1, f\right) .
\end{aligned}
$$


Thus from (8), (9) and (10) we have outside $H$ that

whence

$$
T(r, g)<3 T\left(\left(1+r^{2}\right)^{1 / 2}, f\right)+O(\log r+\log T(r, g))
$$

$$
T(r, g)<4 T\left(r+r^{-1}, f\right)
$$

outside a set $H^{\prime}$ of finite measure.

By Lemma $3 T(r, f)<,\exp (4 \pi r)$ for large $r$, so applying Lemma 4 with $V(r)=T(r, f)(11)$ gives

$$
T(r, g)<8 T(r, f)
$$

outside a set $E_{1}$ in $[1, \infty)$, of finite logarithmic measure.

Lemma 6. (Cf. [5].) Suppose $h$ is entire of infinite order with zeros restricted to a finite number of rays through the origin. Then there exists a set $G \subset[1, \infty)$ having logarithmic density zero and such that $\lim N(r, 0) / T(r, h)=0$ as $r \rightarrow \infty$ outside $G$.

Final section of the proof of the theorem. Let $f$ satisfy the assumptions of the theorem and consider $h(z)=f(-i z)$ which has the same characteristic as $f$, while the zeros and ones of $h$ lie on the lines $\operatorname{Im} z=0$ and $\operatorname{Im} z=1$ respectively.

Suppose that the order of $f$ (and hence of $h$ ) is infinite. Then by Lemma 6 there is a set $G_{1} \subset[1, \infty)$ of logarithmic density zero and such that

$$
\lim N(r, 0, h) / T(r, h)=0 \quad \text { as } \quad r \rightarrow \infty, \quad r \notin G_{1} .
$$

Now consider $g(z)=h(i+z)-1$ which is also of infinite order with real zeros. By Lemma 5 there is a set $G_{2} \subset[1, \cdots)$ of finite logarithmic measure such that $T(r, g)<8 T(r, h), r \notin G_{2}$.

Since $n(r, 1, h) \leqq n(r, 0, g)$ holds we have

$$
N(r, 1, h) \leqq N(r, 0, g)+O(\log r)
$$

and for $r \notin G_{2}$

$$
\frac{N(r, 1, h)}{T(r, h)} \leqq \frac{8 N(r, 0, g)}{T(r, g)}+o(1)
$$

Applying Lemma 6 to $g$ shows that there is a set $G_{3}$ of logarithmic density zero such that as $r \rightarrow \infty$ outside $G_{3}$ the right hand side of (13) tends to zero.

The second fundamental theorem shows that

$$
(1+o(1)) T(r, h) \leqq N(r, 0, h)+N(r, 1, h)
$$

outside a set $G_{4}$ of finite measure. Thus $G=G_{1} \cup G_{2} \cup G_{3} \cup G_{4}$ has zero logarithmic density and as $r \rightarrow \infty$ outside $G$ we have by (12), (13) and (14) that

$$
(1+o(1)) T(r, h)=o(T(r, h))
$$

which is a contradiction. The proof is now complete. 


\section{References}

[1] Borel, E.: Leçons sur les fonctions entières. 2ième éd. - Paris, Gauthier-Villars et $C^{i e}$, Éditeurs, 1921.

[2] Kobayashi, T.: On a characteristic property of the exponential function. - Kōdai Math. Sem. Rep. 29, 1977, 130-156.

[3] Kobayashi, T.: Entire functions with three linearly distributed values. - Kōdai Math. J. 1, 1978, 133-158.

[4] Kobayashi, T.: An entire function with linearly distributed values. - Kōdai Math. J. 2, 1979, 54-81.

[5] Miles, J.: On entire functions of infinite order with radially distributed zeros. - Pacific J. Math. 81, 1979, 131-157.

Imperial College

Department of Mathematics

London S. W. 7

England

Received 25 February 1980 